\title{
Linewidths of collective excitations of the inhomogeneous electron gas: Application to two-dimensional quantum strips
}

\author{
C. A. Ullrich and G. Vignale \\ Department of Physics, University of Missouri, Columbia, Missouri 65211
}

(Received 15 January 1998; revised manuscript received 21 April 1998)

\begin{abstract}
It is well known that high-frequency collective excitations in electronic systems are not Landau damped, i.e., they cannot decay effectively into single particle-hole pairs. The leading damping mechanism in this regime is instead provided by dynamical exchange and correlation effects, such as multipair production. These effects are not captured by the widely used adiabatic local-density approximation (ALDA), which accounts for Landau damping only. In the recently developed time-dependent current density-functional formalism [G. Vignale, C. A. Ullrich, and S. Conti, Phys. Rev. Lett. 79, 4878 (1997)], exchange and correlation enter as viscoelastic stresses in the electron fluid, causing an additional damping that is not contained in the ALDA. We use this theory to derive an explicit formula for the linewidth of collective electronic excitations that are not Landau damped. The formula is then applied to calculate the linewidth of collective modes in two-dimensional (2D) quantum strips. In comparison with the corresponding modes in the homogeneous $2 \mathrm{D}$ electron gas, we find an order-of-magnitude enhancement of the linewidth due to the nonuniformity of the system.
\end{abstract}

[S0163-1829(98)05436-8]

\section{INTRODUCTION}

Collective excitations of electrons in semiconductor nanostructures such as quantum wells, quantum wires, and quantum dots have become a research field of great theoretical and experimental interest in recent years. ${ }^{1}$ This is largely due to the tremendous improvements in semiconductor growth techniques that allow one to tailor the potential across an electron film in a heterostructure practically to any desired shape, through a suitable choice of the quantum well design. As a typical example we mention the widely studied parabolic quantum wells with and without imperfections. ${ }^{2}$ These systems approximate the jellium model much more closely than conventional metals, since their Fermi wavelength is substantially larger than the crystalline lattice spacings, suppressing complicated effects of the lattice potential.

Single ${ }^{3}$ and double ${ }^{4}$ square wells represent another type of heterostructure that is of great theoretical as well as technological interest. The lowest collective intersubband excitations in these systems have energies below the optical phonon energy and have been studied in photoabsorption experiments in the far-infrared regime. The energy relaxation for these modes proceeds via electron-electron, electronimpurity, and acoustical-phonon scattering, as well as optical-phonon scattering (at nonzero temperature). However, the relative importance of the various possible dissipation mechanisms is still not well understood.

In this work, we consider the decay of collective excitations in inhomogeneous electronic systems due to electronelectron interaction, which is the leading effect at zero temperature and in the absence of impurities. The main damping mechanism of low-frequency collective electronic modes is decay into single particle-hole pairs, also known as Landau damping. ${ }^{5}$ For high-frequency modes, however, this decay mechanism is not effective, and damping is instead induced by dynamical exchange and correlation (xc) effects such as multipair production. In the following, we shall develop a new method to calculate the linewidth of collective excitations related to dynamical xc effects.

The standard method for calculating collective excitations of the inhomogeneous electron gas is to find the poles of the density-density response function. ${ }^{5,6}$ Such a calculation is conveniently performed within the framework of timedependent density-functional theory. ${ }^{7,8}$ In this formalism, all dynamic xc effects of the electron gas are accounted for through a local time-dependent xc potential $v_{\mathrm{xc}}(\mathbf{r}, t)$. The latter is usually approximated as a function of the instantaneous local density,

$$
v_{\mathrm{xc}}^{\mathrm{ALDA}}(\mathbf{r}, t)=\left.\frac{d e_{\mathrm{xc}}(\varrho)}{d \varrho}\right|_{\varrho=\varrho(\mathbf{r}, t)},
$$

where $e_{\mathrm{xc}}(\varrho)$ is the xc energy density of the homogeneous electron gas of density $\varrho$. This scheme is known as the adiabatic local density approximation (ALDA). If used to calculate the full response function of the system, the ALDA accounts for the Landau damping of high-frequency collective modes. However, outside the regime where Landau damping occurs, the modes calculated within the ALDA will come out undamped. ${ }^{9,10}$ To describe the relaxation of these modes over the whole range of frequencies and wave vectors, one has to go beyond the ALDA and include retardation effects. This task can be accomplished with the use of the recently developed time-dependent current density-functional theory. ${ }^{11,12}$ In this formalism, dynamical exchange and correlation lead to the appearance of viscoelastic stresses in the electron fluid, ${ }^{13}$ with complex and frequency-dependent viscosity coefficients depending on properties of the homogeneous electron gas. The viscosity, in particular, causes an additional damping not contained in the ALDA.

The paper is organized as follows: In Sec. II we briefly review the main features of time-dependent current densityfunctional theory in the linear regime. We then derive in Sec. 
III a formula to calculate the linewidth of collective excitations outside the regime of Landau damping. The idea is to first calculate the (undamped) modes within the ALDA and then include the viscosity-induced damping perturbatively. Relevant details of the calculation are given in the Appendix. In order to demonstrate the practicality of the approach, we then apply it in Sec. IV to collective modes in twodimensional (2D) quantum strips. ${ }^{14}$ The advantage of these systems is that they can be treated analytically, within a hydrodynamical approximation, and thus allow us to gain systematic insight into the damping of collective modes in an inhomogeneous model system. We find that the boundary effects caused by the confinement of the 2D electron gas (2DEG) lead to a strong enhancement of the linewidth as compared to the homogeneous case. A detailed comparison of our theory with experimental results, which are available for systems such as the quantum wells mentioned above, is the subject of currently ongoing studies.

\section{CURRENT DENSITY-FUNCTIONAL THEORY IN THE LINEAR REGIME}

We consider an interacting electronic system with ground-state density $\varrho_{0}(\mathbf{r})$ that is perturbed by a weak external vector potential $\mathbf{a}_{1}(\mathbf{r}, \omega) e^{-i \omega t}$. The current-density response $\mathbf{j}_{1}(\mathbf{r}, \omega) e^{-i \omega t}$, to first order in $\mathbf{a}_{1}$, is given by

$$
j_{1, \mu}(\mathbf{r}, \omega)=\sum_{\nu} \int d^{3} r^{\prime} \chi_{\mathrm{KS}, \mu \nu}\left(\mathbf{r}, \mathbf{r}^{\prime}, \omega\right) a_{\mathrm{eff1} 1, \nu}\left(\mathbf{r}^{\prime}, \omega\right) .
$$

Here, $\chi_{\mathrm{KS}, \mu \nu}\left(\mathbf{r}, \mathbf{r}^{\prime}, \omega\right)$ is the current-current response function of a system of noninteracting electrons with the same density $\varrho_{0}(\mathbf{r})$, defined as

$$
\begin{aligned}
\chi_{\mathrm{KS}, \mu \nu}\left(\mathbf{r}, \mathbf{r}^{\prime}, \omega\right)= & \frac{\varrho_{0}(\mathbf{r})}{m} \delta\left(\mathbf{r}-\mathbf{r}^{\prime}\right) \delta_{\mu \nu}+\frac{1}{m^{2}} \sum_{\alpha, \beta}\left(f_{\alpha}-f_{\beta}\right) \\
& \times \frac{\psi_{\alpha}^{*}(\mathbf{r}) \nabla_{\mu} \psi_{\beta}(\mathbf{r}) \psi_{\beta}^{*}\left(\mathbf{r}^{\prime}\right) \nabla_{\nu}^{\prime} \psi_{\alpha}\left(\mathbf{r}^{\prime}\right)}{\omega-\left(\varepsilon_{\beta}-\varepsilon_{\alpha}\right)+i \eta},
\end{aligned}
$$

where $\psi_{\alpha}$ are the solutions of the unperturbed static KohnSham equation, with eigenvalues $\varepsilon_{\alpha}$ (note that we set $e=c$ $=1$ in this and the following section). The effective vector potential entering Eq. (2) is

$$
\begin{aligned}
\mathbf{a}_{\mathrm{eff} 1}(\mathbf{r}, \omega)= & \mathbf{a}_{1}(\mathbf{r}, \omega)+\frac{\nabla}{(i \omega)^{2}} \int d^{3} r^{\prime} \frac{\nabla^{\prime} \cdot \mathbf{j}_{1}\left(\mathbf{r}^{\prime}, \omega\right)}{\left|\mathbf{r}-\mathbf{r}^{\prime}\right|} \\
& +\mathbf{a}_{\mathrm{xc1}}(\mathbf{r}, \omega) .
\end{aligned}
$$

$\mathbf{a}_{\mathrm{xc} 1}(\mathbf{r}, \omega)$ is the first-order xc vector potential (which in general contains both longitudinal and transverse terms). It may be expressed as

$$
\mathbf{a}_{\mathrm{xcl}}(\mathbf{r}, \omega)=\int d^{3} r^{\prime} \mathbf{f}_{\mathrm{xc}}\left(\mathbf{r}, \mathbf{r}^{\prime}, \omega\right) \cdot \mathbf{j}_{1}\left(\mathbf{r}^{\prime}, \omega\right),
$$

where the $3 \times 3$ tensor kernel $f_{\mathrm{xc}, \mu \nu}$ is defined as

$$
\begin{aligned}
f_{\mathrm{xc}, \mu \nu}\left(\mathbf{r}, \mathbf{r}^{\prime}, \omega\right)= & \chi_{\mathrm{KS}, \mu \nu}^{-1}\left(\mathbf{r}, \mathbf{r}^{\prime}, \omega\right)-\chi_{\mu \nu}^{-1}\left(\mathbf{r}, \mathbf{r}^{\prime}, \omega\right) \\
& -\frac{1}{(i \omega)^{2}} \nabla_{\mu} \frac{1}{\left|\mathbf{r}-\mathbf{r}^{\prime}\right|} \nabla_{\nu}^{\prime}
\end{aligned}
$$

( $\chi_{\mu \nu}$ is the full response function of the interacting system).

A local-density approximation for $\mathbf{a}_{\mathrm{xcl}}(\mathbf{r}, \omega)$ has recently been derived by Vignale and Kohn. ${ }^{11}$ Their expression becomes exact when the equilibrium density and external potential are slowly varying in space, on length scales set by $k_{F}^{-1}$ and $v_{F} / \omega$, where $k_{F}$ and $v_{F}$ are, respectively, the local Fermi wave number and velocity. It can be written in the following form: ${ }^{13}$

$i \omega a_{\mathrm{xc} 1, \mu}(\mathbf{r}, \omega)=\nabla_{\mu} v_{\mathrm{xc} 1}^{\mathrm{ALAA}}(\mathbf{r}, \omega)-\frac{1}{\varrho_{0}(\mathbf{r})} \sum_{\nu} \nabla_{\nu} \sigma_{\mathrm{xc}, \mu \nu}(\mathbf{r}, \omega)$.

The first term is the linearized ALDA expression (1), and the dynamical correction is the divergence of the viscoelastic stress tensor

$$
\sigma_{\mathrm{xc}, \mu \nu}=\eta_{\mathrm{xc}}\left(\nabla_{\nu} u_{\mu}+\nabla_{\mu} u_{\nu}-\frac{2}{3} \nabla \cdot \mathbf{u} \delta_{\mu \nu}\right)+\zeta_{\mathrm{xc}} \nabla \cdot \mathbf{u} \delta_{\mu \nu}
$$

Here, $\mathbf{u}(\mathbf{r}, \omega)=\mathbf{j}_{1}(\mathbf{r}, \omega) / \varrho_{0}(\mathbf{r})$ is the velocity field, and $\eta_{\mathrm{xc}}\left(\omega, \varrho_{0}(\mathbf{r})\right)$ and $\zeta_{\mathrm{xc}}\left(\omega, \varrho_{0}(\mathbf{r})\right)$ are complex viscosity coefficients. They are related to the longitudinal and transverse homogeneous electron gas functions $f_{\mathrm{xcL}}^{h}(\omega, \varrho)$ and $f_{\mathrm{xcT}}^{h}(\omega, \varrho)$ :

$$
\begin{gathered}
\eta_{\mathrm{xc}}(\omega, \varrho)=-\frac{\varrho^{2}}{i \omega} f_{\mathrm{xcT}}^{h}, \\
\zeta_{\mathrm{xc}}(\omega, \varrho)=-\frac{\varrho^{2}}{i \omega}\left[f_{\mathrm{xcL}}^{h}-\frac{4}{3} f_{\mathrm{xcT}}^{h}-\frac{d^{2} e_{\mathrm{xc}}(\varrho)}{d \varrho^{2}}\right] .
\end{gathered}
$$

The functions $f_{\mathrm{xcL}(\mathrm{T})}^{h}$ are defined in terms of the dynamic local field factors ${ }^{15} \quad G_{\mathrm{L}(\mathrm{T})}(k, \omega)$ as $f_{\mathrm{xcL}(\mathrm{T})}^{h}=$ $-\lim _{k \rightarrow 0} 4 \pi G_{\mathrm{L}(\mathrm{T})}(k, \omega) / k^{2}$. Explicit parametrizations of the frequency-dependent $f_{\mathrm{xcL}(\mathrm{T})}^{h}$ have recently been calculated in 2D and 3D by Nifosi, Conti, and Tosi ${ }^{16,17}$ using an approximate decoupling scheme of the equation of motion for the current density, which accounts for processes of excitation of two electron-hole pairs.

\section{LINEWIDTH OF COLLECTIVE EXCITATIONS}

The collective modes of an electronic system can be found by determining the poles $\Omega_{n}$ of the full response function in the complex $\omega$ plane. In this special case, the response equation (2) will have finite solutions if the external perturbation is set to zero in Eq. (4). One has to solve the integral equation

$$
\begin{aligned}
\sum_{\nu} \int & d^{3} r^{\prime}\left[\chi_{\mathrm{ALDA}, \mu \nu}^{-1}\left(\mathbf{r}, \mathbf{r}^{\prime}, \Omega_{n}\right)\right. \\
& \left.-f_{\mathrm{xc}, \mu \nu}^{\mathrm{visc}}\left(\mathbf{r}, \mathbf{r}^{\prime}, \Omega_{n}\right)\right] J_{n, \nu}\left(\mathbf{r}^{\prime}, \Omega_{n}\right)=0
\end{aligned}
$$


to determine the collective mode frequencies $\Omega_{n}$ and associated mode profiles $\mathbf{J}_{n}\left(\mathbf{r}, \Omega_{n}\right)$. Here, the ALDA inverse response function is given by

$$
\begin{aligned}
\chi_{\mathrm{ALDA}, \mu \nu}^{-1}\left(\mathbf{r}, \mathbf{r}^{\prime}, \omega\right)= & \chi_{\mathrm{KS}, \mu \nu}^{-1}\left(\mathbf{r}, \mathbf{r}^{\prime}, \omega\right)+\frac{\nabla_{\mu}}{\omega^{2}}\left[\frac{1}{\left|\mathbf{r}-\mathbf{r}^{\prime}\right|}\right. \\
& +\left.\delta\left(\mathbf{r}-\mathbf{r}^{\prime}\right) \frac{d^{2} e_{\mathrm{xc}}(\varrho)}{d \varrho^{2}}\right|_{\varrho=\varrho_{0}(\mathbf{r})} \nabla_{\nu}^{\prime},
\end{aligned}
$$

and the viscosity term $f_{\mathrm{xc}, \mu \nu}^{\mathrm{visc}}$ is defined in terms of the stress tensor (8) as

$$
\begin{gathered}
\sum_{\nu} \int d^{3} r^{\prime} f_{\mathrm{xc}, \mu \nu}^{\mathrm{visc}}\left(\mathbf{r}, \mathbf{r}^{\prime}, \omega\right) j_{\nu}\left(\mathbf{r}^{\prime}, \omega\right) \\
=\frac{i}{\varrho_{0}(\mathbf{r}) \omega} \sum_{\nu} \nabla_{\nu} \sigma_{\mathrm{xc}, \mu \nu}(\mathbf{r}, \omega)
\end{gathered}
$$

The next step is to multiply Eq. (11) from the right with $J_{n, \mu}^{*}\left(\mathbf{r}, \Omega_{n}\right)$ and integrate over $\mathbf{r}$ :

$$
\begin{aligned}
\sum_{\mu \nu} \int & d^{3} r \int d^{3} r^{\prime}\left[\chi_{\mathrm{ALDA}, \mu \nu}^{-1}\left(\mathbf{r}, \mathbf{r}^{\prime}, \Omega_{n}\right)\right. \\
& \left.-f_{\mathrm{xc}, \mu \nu}^{\mathrm{visc}}\left(\mathbf{r}, \mathbf{r}^{\prime}, \Omega_{n}\right)\right] J_{n, \nu}\left(\mathbf{r}^{\prime}, \Omega_{n}\right) J_{n, \mu}^{*}\left(\mathbf{r}, \Omega_{n}\right)=0 .
\end{aligned}
$$

In the following, we restrict ourselves to the case where the only effective damping occurs via dynamical xc effects, as discussed in the introduction. We further assume that the damping is only a small effect, i.e., $\operatorname{Re} \Omega_{n} \gg\left|\operatorname{Im} \Omega_{n}\right|$. In other words, the viscosity term in Eq. (11) can be viewed as a small perturbation to the ALDA response function (12). This means that if we solve the (undamped) ALDA response equation

$$
\sum_{\nu} \int d^{3} r^{\prime} \chi_{\mathrm{ALDA}, \mu \nu}^{-1}\left(\mathbf{r}, \mathbf{r}^{\prime}, \omega_{n}\right) j_{n, \nu}\left(\mathbf{r}^{\prime}, \omega_{n}\right)=0
$$

(where $\omega_{n}$ is real), we expect the resulting $\omega_{n}$ and $j_{n, \nu}\left(\mathbf{r}, \omega_{n}\right)$ to be close to $\operatorname{Re} \Omega_{n}$ and $J_{n, \nu}\left(\mathbf{r}, \Omega_{n}\right)$, obtained from solving the full response equation (11). We can then perform an expansion of $J_{n, \nu}\left(\mathbf{r}^{\prime}, \Omega_{n}\right)$ around the undamped mode frequency $\omega_{n}$ :

$$
J_{n, \nu}\left(\mathbf{r}^{\prime}, \Omega_{n}\right) \approx j_{n, \nu}\left(\mathbf{r}^{\prime}, \omega_{n}\right)+\left.\left(\Omega_{n}-\omega_{n}\right) \frac{\partial j_{n, \nu}\left(\mathbf{r}^{\prime}, \xi\right)}{\partial \xi}\right|_{\xi=\omega_{n}}
$$

and similarly for $J_{n, \mu}^{*}\left(\mathbf{r}, \Omega_{n}\right), \quad \chi_{\mathrm{ALDA}, \mu \nu}^{-1}\left(\mathbf{r}, \mathbf{r}^{\prime}, \Omega_{n}\right)$ and $f_{\mathrm{xc}, \mu \nu}^{\mathrm{visc}}\left(\mathbf{r}, \mathbf{r}^{\prime}, \Omega_{n}\right)$. Introducing these expansions into Eq. (14), we get

$$
\begin{aligned}
\sum_{\mu \nu} & \int d^{3} r \int d^{3} r^{\prime}\left[\left(\Omega_{n}-\omega_{n}\right) \frac{\partial}{\partial \omega_{n}} \chi_{\mathrm{ALDA}, \mu \nu}^{-1}\left(\mathbf{r}, \mathbf{r}^{\prime}, \omega_{n}\right)\right. \\
& \left.-f_{\mathrm{xc}, \mu \nu}^{\mathrm{visc}}\left(\mathbf{r}, \mathbf{r}^{\prime}, \omega_{n}\right)\right] \\
& \times j_{n, \nu}\left(\mathbf{r}^{\prime}, \omega_{n}\right) j_{n, \mu}^{*}\left(\mathbf{r}, \omega_{n}\right)=0 .
\end{aligned}
$$

To arrive at this relation, we dropped terms of second order in $\left(\Omega_{n}-\omega_{n}\right)$ and $f_{\mathrm{xc}, \mu \nu}^{\mathrm{visc}}$, and made use of Eq. (11). We thus obtain the viscosity-induced frequency shift as

$$
\Omega_{n}-\omega_{n}=\frac{\sum_{\mu \nu} \int d^{3} r \int d^{3} r^{\prime} f_{\mathrm{xc}, \mu \nu}^{\mathrm{visc}}\left(\mathbf{r}, \mathbf{r}^{\prime}, \omega_{n}\right) j_{n, \nu}\left(\mathbf{r}^{\prime}, \omega_{n}\right) j_{n, \mu}^{*}\left(\mathbf{r}, \omega_{n}\right)}{\sum_{\mu \nu} \int d^{3} r \int d^{3} r^{\prime}\left\{\left(\partial / \partial \omega_{n}\right) \chi_{\mathrm{ALDA}, \mu \nu}^{-1}\left(\mathbf{r}, \mathbf{r}^{\prime}, \omega_{n}\right)\right\} j_{n, \nu}\left(\mathbf{r}^{\prime}, \omega_{n}\right) j_{n, \mu}^{*}\left(\mathbf{r}, \omega_{n}\right)} .
$$

To simplify this expression, we rewrite the numerator using definition (13). For the denominator, we employ an explicit expression for $\chi_{\mathrm{KS}, \mu \nu}^{-1}\left(\mathbf{r}, \mathbf{r}^{\prime}, \omega\right)$, valid for small density variation, which is derived in the Appendix, see Eq. (A13). Using this approximation, $\chi_{\mathrm{ALDA}, \mu \nu}^{-1}\left(\mathbf{r}, \mathbf{r}^{\prime}, \omega\right)$ is explicitly known and real, and we can easily calculate its derivative with respect to $\omega$ :

$$
\begin{aligned}
& \frac{\partial}{\partial \omega} \chi_{\mathrm{ALDA}, \mu \nu}^{-1}\left(\mathbf{r}, \mathbf{r}^{\prime}, \omega\right) \\
& \quad=-\frac{2}{\omega}\left[\chi_{\mathrm{ALDA}, \mu \nu}^{-1}\left(\mathbf{r}, \mathbf{r}^{\prime}, \omega\right)-\frac{m}{\varrho_{0}(\mathbf{r})} \delta_{\mu \nu} \delta\left(\mathbf{r}-\mathbf{r}^{\prime}\right)\right]
\end{aligned}
$$

Using Eq. (11) and introducing the velocity field $u_{n, \mu}$ $=j_{n, \mu} / \varrho_{0}$, we thus end up with

$$
\Omega_{n}-\omega_{n}=\frac{i}{2 m} \frac{\sum_{\mu \nu} \int d^{3} r u_{n, \mu}^{*}\left(\mathbf{r}, \omega_{n}\right) \nabla_{\nu} \sigma_{\mathrm{xc}, \mu \nu}\left(\mathbf{r}, \omega_{n}\right)}{\int d^{3} r \varrho_{0}(\mathbf{r})\left|\mathbf{u}_{n}\left(\mathbf{r}, \omega_{n}\right)\right|^{2}} .
$$

$\Omega_{n}-\omega_{n}$ is in general a complex quantity. The imaginary part of it leads to a broadening of the intensity profile of the collective mode. To be precise, the $\delta$ shape obtained within the ALDA is converted into a Lorentzian with FWHM (full width at half-maximum) $\Gamma_{n}$, where

$$
\Gamma_{n}=\frac{\left|\operatorname{Re} \Sigma_{\mu \nu} \int d^{3} r u_{n, \mu}^{*}\left(\mathbf{r}, \omega_{n}\right) \nabla_{\nu} \sigma_{\mathrm{xc}, \mu \nu}\left(\mathbf{r}, \omega_{n}\right)\right|}{m \int d^{3} r \varrho_{0}(\mathbf{r})\left|\mathbf{u}_{n}\left(\mathbf{r}, \omega_{n}\right)\right|^{2}}
$$

The linewidth formula (21) has a very simple physical interpretation that clearly shows the analogy with classical hydrodynamics. ${ }^{18}$ It can be written as 


$$
\Gamma_{n}=\frac{\left|\bar{E}^{\prime}\right|}{\bar{E}}
$$

where

$$
\bar{E}^{\prime}=2 \operatorname{Re} \sum_{\mu \nu} \int d^{3} r u_{n, \mu}^{*}\left(\mathbf{r}, \omega_{n}\right) \nabla_{\nu} \sigma_{\mathrm{xc}, \mu \nu}\left(\mathbf{r}, \omega_{n}\right)
$$

is the average energy dissipated per unit time by the viscosity, and

$$
\bar{E}=2 m \int d^{3} r \varrho_{0}(\mathbf{r})\left|\mathbf{u}_{n}\left(\mathbf{r}, \omega_{n}\right)\right|^{2}
$$

is the mechanical energy stored in the oscillation of the electron fluid, regarded as slowly decaying in time.

\section{COLLECTIVE MODES IN QUANTUM STRIPS}

\section{A. Solution of the linearized classical equation of motion}

We consider in the following a single quantum strip or wire of width $2 W$ and infinite length, defined in the $y z$ plane of a 2DEG by the application of a parabolic confining potential $v=m^{*} \Omega^{2} y^{2} / 2$, where $m^{*}$ is the electron effective mass. The strip is oriented along the $z$ axis, and we assume the density to be uniform in this direction. The collective modes in this system have been studied ${ }^{14,19}$ using a classical approach (which does not include damping), and we here briefly summarize the results. The classical equation of motion for the velocity field $u(y, t)$ of the $2 \mathrm{D}$ electron fluid (without external driving field) reads

$$
m^{*} \frac{\partial u(y, t)}{\partial t}=-m^{*} \Omega^{2} y+2 e^{* 2} \int d y^{\prime} \frac{\varrho\left(y^{\prime}, t\right)}{y-y^{\prime}} .
$$

Here, $e^{* 2}=e^{2} / \epsilon$ accounts for the screening of the electronic charge $e$ by a background with dielectric constant $\epsilon$, and $\varrho(y, t)$ is the $2 \mathrm{D}$ number density. The leading corrections to this equation of motion arising from kinetic and xc energy can be shown ${ }^{19}$ to be negligible compared to the classical electrostatic energy as long as $W \gg a_{0}^{*}$, where $a_{0}^{*}$ $=\hbar^{2} / m^{*} e^{* 2}$ is the effective Bohr radius. This condition is safely fulfilled for the systems we are going to treat here.

The equilibrium density distribution $\varrho_{0}(y)$ of the quantum strip is given by

$$
\varrho_{0}(y)=\frac{2 \lambda}{\pi W} \sqrt{1-\tilde{y}^{2}}, \quad|y| \leqslant W .
$$

Here, $\tilde{y}=y / W, \lambda$ is the number of electrons per unit length in $z$ direction, and

$$
\Omega^{2}=\frac{4 \lambda e^{* 2}}{m^{*} W^{2}}=\varrho_{0}(0) \frac{2 \pi e^{* 2}}{m^{*} W} .
$$

To determine the collective modes, Eq. (25) is linearized about the equilibrium density distribution (26):

$$
\varrho(y, t)=\varrho_{0}(y)+\varrho_{1}(y) e^{-i \omega t} .
$$

Furthermore, the linearized continuity equation

$$
-i \omega \varrho_{1}+\frac{\partial}{\partial y}\left(\varrho_{0} u_{1}\right)=0
$$

is used, where it is assumed that $u_{1}(y, t)$ varies as $e^{-i \omega t}$. In this fashion, the $n$th normal mode in the quantum strip is found to be $\mathrm{e}^{14}$

$$
\varrho_{1, n}(y)=\eta_{n} \frac{T_{n}(\tilde{y})}{\sqrt{1-\tilde{y}^{2}}}, \quad n=1,2,3, \ldots,
$$

where $T_{n}$ denotes Chebyshev polynomials of the first kind, ${ }^{20}$ and $\eta_{n}$ is a normalization constant which has the dimensions of a $2 \mathrm{D}$ density. The associated mode frequencies are given by

$$
\omega_{n}^{2}=n \Omega^{2}=\frac{2 \pi \varrho_{0}(0) e^{* 2}}{m^{*}} \frac{n}{W} .
$$

The velocity profile $u_{1, n}$ of the $n$th mode is obtained in a straightforward manner from the continuity equation (29). We make use of the connection

$$
\frac{d}{d \tilde{y}}\left[\sqrt{1-\tilde{y}^{2}} U_{n-1}(\tilde{y})\right]=-n \frac{T_{n}(\tilde{y})}{\sqrt{1-\tilde{y}^{2}}}
$$

between the Chebyshev polynomials of the first and second kind, ${ }^{20}$ and we end up with

$$
u_{1, n}\left(y, \omega_{n}\right)=-\frac{i \omega_{n} W \eta_{n}}{\varrho_{0}(0) n} U_{n-1}(\tilde{y}) .
$$

The first mode, $u_{1,1}\left(y, \omega_{1}\right)$, is the well-known center-of-mass mode with $\omega_{1}=\Omega$. Since $U_{0}(\tilde{y})=1$, it has a uniform velocity profile.

\section{B. Calculation of the linewidth}

Let us now insert the collective modes of the 2D quantum strip into the linewidth formula (21). First of all, with Eqs. (26) and (33) the denominator becomes

$$
\begin{aligned}
& m^{*} \int d^{3} r \varrho_{0}(\mathbf{r})\left|\mathbf{u}_{1, n}\left(\mathbf{r}, \omega_{n}\right)\right|^{2} \\
& \quad=m^{*} L \frac{\omega_{n}^{2} W^{3} \eta_{n}^{2}}{\varrho_{0}(0) n^{2}} \int_{-1}^{1} d \tilde{y} \sqrt{1-\tilde{y}^{2}} U_{n-1}^{2}(\tilde{y}),
\end{aligned}
$$

where $L$ denotes the length of the strip (we assume $L \rightarrow \infty$ ). Due to the orthogonality of the Chebyshev polynomials, ${ }^{20}$ the integral on the right-hand side yields a value of $\pi / 2$.

Next, consider the stress tensor (8), which in two dimensions reduces to

$$
\begin{aligned}
\sigma_{\mathrm{xc}, \mu \nu}^{2 \mathrm{D}}= & \eta_{\mathrm{xc}}\left(\nabla_{\nu} u_{\mu}+\nabla_{\mu} u_{\nu}-\nabla \cdot \mathbf{u} \delta_{\mu \nu}\right) \\
& +\zeta_{\mathrm{xc}} \nabla \cdot \mathbf{u} \delta_{\mu \nu},
\end{aligned}
$$

so that 


$$
\begin{aligned}
\sum_{\mu \nu} \int & d^{3} r u_{n, \mu}^{*} \nabla_{\nu} \sigma_{\mathrm{xc}, \mu \nu}^{2 \mathrm{D}} \\
= & L \frac{\omega_{n}^{2} W \eta_{n}^{2}}{\varrho_{0}(0)^{2} n^{2}} \int_{-1}^{1} d \tilde{y} U_{n-1}(\tilde{y}) \frac{\partial}{\partial \tilde{y}} \\
& \times\left[\left(\zeta_{\mathrm{xc}}+\eta_{\mathrm{xc}}\right) \frac{\partial U_{n-1}(\tilde{y})}{\partial \tilde{y}}\right] .
\end{aligned}
$$

From Eqs. (9) and (10) it follows that $\operatorname{Re}\left(\zeta_{\mathrm{xc}}+\eta_{\mathrm{xc}}\right)=$ $-\left(\varrho_{0}^{2} / \omega\right) \operatorname{Im} f_{\mathrm{xcL}}^{h}\left(\varrho_{0}, \omega\right)$ (it can be shown that this relation is valid both in $2 \mathrm{D}$ and in $3 \mathrm{D})$. Thus, performing a partial integration in Eq. (36) and dividing by Eq. (34), we get the ratio between linewidth and mode frequency of the $n$th collective mode in the quantum strip:

$$
\begin{aligned}
\frac{\Gamma_{n}}{\omega_{n}}= & \frac{n}{\pi^{2} e^{* 2} W} \int_{-1}^{1} d \tilde{y}\left(1-\tilde{y}^{2}\right) \\
& \times\left|\operatorname{Im} f_{\mathrm{xcL}}^{h}\left(\varrho_{0}(\tilde{y}), \omega_{n}\right)\right|\left(\frac{1}{n} \frac{\partial U_{n-1}(\tilde{y})}{\partial \tilde{y}}\right)^{2} .
\end{aligned}
$$

We see immediately that the center-of-mass mode $(n=1)$ is undamped since $\partial U_{0} / \partial \tilde{y}=0$. All modes with $n \geqslant 2$ will have a nonvanishing linewidth, however.

By construction, Eq. (37) is appropriate for modes with small index $n$, which are the most important cases in practice. However, the situation gets more complicated as the mode index $n$ increases $(n \gtrsim 10)$. The Chebyshev polynomials $U_{n-1}(\tilde{y})$ then become more and more rapidly oscillating, especially towards the edges of the strip. In the limit of $n, W \rightarrow \infty$ for fixed ratio $n / W$, application of Eq. (37) as it stands would lead to a logarithmically diverging linewidth. The reason for this clearly unphysical behavior is that the hydrodynamical modes, which we take as unperturbed solutions for our linewidth formula, are well defined only when they are slowly varying. In reality, for large $n$ the modes enter the regime where Landau damping (creation of single electron-hole pairs) becomes possible, which will suppress the short-wavelength components of the mode. In order to account for this effect, the formalism to calculate the linewidth will be suitably modified in the following.

\section{Landau versus xc damping}

Let us first consider the case of a homogeneous 2DEG of density $\bar{\varrho}=1 / \pi r_{s}^{2}$ and Fermi wave vector $\bar{q}_{F}=\sqrt{2 \pi \bar{\varrho}}$. Landau damping sets in when the plasmon dispersion curve $\omega_{\text {2DEG }}(q)$ (see below) enters the particle-hole continuum. This occurs at a certain cutoff wave vector $\bar{q}_{c}$, which may be determined by solving ${ }^{21}$

$$
\omega_{2 \mathrm{DEG}}\left(\bar{q}_{c}\right)=\bar{q}_{c} \bar{q}_{F}+\frac{\bar{q}_{c}^{2}}{2} .
$$

In order to obtain a comparable criterion for the inhomogeneous quantum strips, we need to introduce the concept of the local wave vector $q_{n}(\tilde{y})$. We first consider the center of the strip where $\tilde{y} \approx 0$. Making use of the identity ${ }^{20}$

$$
U_{n-1}(\tilde{y})=\frac{\sin (n \arccos \tilde{y})}{\sqrt{1-\tilde{y}^{2}}},
$$

and expanding $\arccos \tilde{\mathrm{y}} \approx(\pi / 2)-\tilde{\mathrm{y}}$ for small $\tilde{y}$, it is easy to see that the normal collective modes (33) reduce for $\tilde{y} \approx 0$ to

$$
u_{1, n}(\tilde{y}) \rightarrow-\frac{i \omega_{n} \eta_{n} W}{\varrho_{0}(0) n}\left\{\begin{array}{lll}
-(-1)^{n / 2} \sin (n \tilde{y}), & n & \text { even } \\
(-1)^{(n-1) / 2} \cos (n \tilde{y}), & n & \text { odd }
\end{array}\right.
$$

We can therefore assign to each collective mode a wave vector $q_{n}(0)=n / W$ in the center of the strip. Assuming that the modes behave locally as $\sin \left[q_{n}(\tilde{y}) y\right]$ or $\cos \left[q_{n}(\tilde{y}) y\right]$ for all values of $|y|<W$, we can determine the local wave vector as $q_{n}(\tilde{y})=\pi /\left[W \Delta_{n}(\tilde{y})\right]$, where $\Delta_{n}(\tilde{y})$ denotes the local distance between two nodes of $u_{1, n}\left(\tilde{y}, \omega_{n}\right)$. To determine $\Delta_{n}(\tilde{y})$, we make use of the fact ${ }^{20}$ that the $m$ th zero of the Chebyshev polynomial $U_{n-1}(\tilde{y})$ is located at the position $\tilde{y}_{m}=\cos [(m / n) \pi]$. Therefore,

$$
\begin{aligned}
\Delta_{n}(\tilde{y}) & =\cos \left(\frac{m}{n} \pi\right)-\cos \left(\frac{m+1}{n} \pi\right) \\
& \approx \frac{\pi}{n} \sin \left(\frac{m}{n} \pi\right)=\frac{\pi}{n} \sqrt{1-\tilde{y}_{m}^{2}},
\end{aligned}
$$

where we assume $n, m \gg 1$. This yields the local wave vector

$$
q_{n}(\tilde{y})=\frac{n}{W} \frac{1}{\sqrt{1-\tilde{y}^{2}}} .
$$

The local cutoff wave vector $q_{c}(\tilde{y})$ now follows from Eq. (38) by replacing the homogeneous density $\bar{\varrho}$ with the equilibrium density profile $\varrho_{0}(\tilde{y})=\varrho_{0}(0) \sqrt{1-\tilde{y}^{2}}$, see Eq. (26), thus defining a local Fermi wave vector $q_{F}(\tilde{y})=\sqrt{2 \pi \varrho_{0}(\tilde{y})}$. We then use relation (31) in place of the plasmon dispersion $\omega_{2 \mathrm{DEG}}(q)$. This yields the following equation for $q_{c}(\tilde{y})$ :

$$
q_{c}(\tilde{y})\left(q_{F}(\tilde{y})+\frac{q_{c}(\tilde{y})}{2}\right)^{2}=2 \pi \varrho_{0}(0) \frac{e^{* 2}}{m^{*}} \sqrt{1-\tilde{y}^{2}} .
$$

To illustrate this concept, we consider the concrete numerical example of a quantum strip with $\varrho_{0}(0)=2.0$ $\times 10^{11} \mathrm{~cm}^{-2}$ and arbitrary width $2 W\left(W \gg a_{0}^{*}\right)$. Having in mind systems based on $\mathrm{GaAs} / \mathrm{Al}_{x} \mathrm{Ga}_{1-x \mathrm{As}}$, we set $m^{*}$ $=0.07 \mathrm{~m}$ and $\epsilon=13$. This means that the Bohr radius becomes $a_{0}^{*}=98.3 \AA$, and we use Hartree energy units with 1 Hartree $^{*}=11.27 \mathrm{meV}$.

In Fig. 1 we plot the local cutoff wave vector $q_{c}(\tilde{y})$ for this strip, together with the local wave vectors $q_{n}(\tilde{y})$ of some collective modes with $q_{n}(0)=n / W=\alpha q_{c}(0)$, where $\alpha$ varies between 0.1 and 0.9 . We see that $q_{n}$ lies well below $q_{c}$ in the interior of the strip as long as $\alpha$ is still small, and $q_{c}(\tilde{y})$ and $q_{n}(\tilde{y})$ intersect at $\tilde{y}_{c}^{(n)}$ close to $\tilde{y}= \pm 1$. For $\alpha \rightarrow 1$, the 


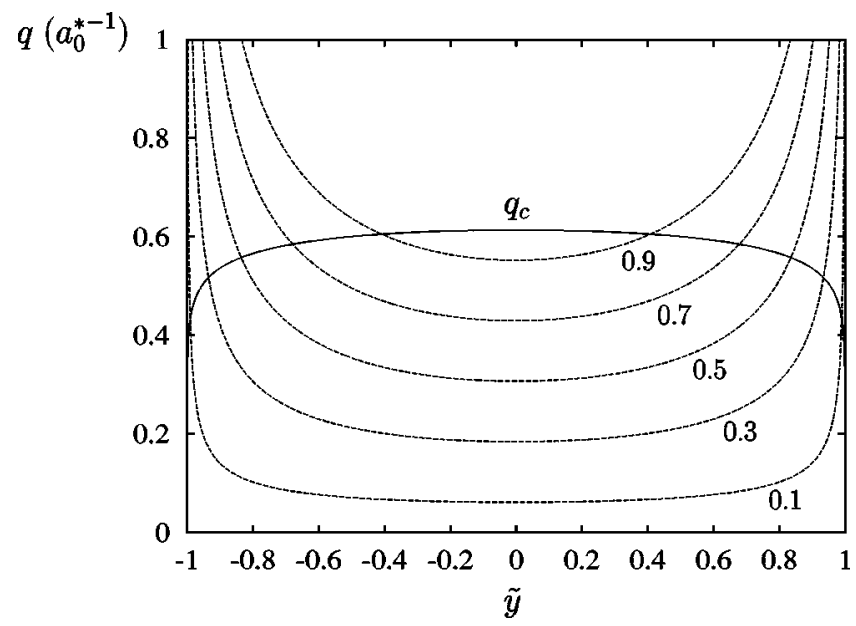

FIG. 1. Full line: cutoff wave vector $q_{c}(\tilde{y})$ [Eq. (43)] for a quantum strip of width $2 \mu \mathrm{m}$ and density in the center $\varrho_{0}(0)=2$ $\times 10^{11} \mathrm{~cm}^{-2}$. Dashed lines: local wave vector $q_{n}(\tilde{y})$ [Eq. (42)] of collective modes with $q_{n}(0)=\alpha q_{c}(0)$, with values of $\alpha$ between 0.1 and 0.9 , as indicated. In the case of large $n$ and $W$, Landau damping occurs mostly in the regions of the strip where $q_{n}>q_{c}$, i.e., between the points of intersection $\tilde{y}_{c}^{(n)}$ and the boundaries of the strip. In the interior region, damping is mainly caused by the much weaker dynamical xc effects. For $q_{n}(0)>q_{c}(0)$, the modes are completely Landau damped.

curves for $q_{n}(\tilde{y})$ move upwards and the points of intersection with $q_{c}(\tilde{y})$ move towards the center of the strip $\left(\left|\tilde{y}_{c}^{(n)}\right|\right.$ $\rightarrow 0)$. For $\alpha>1, q_{n}(\tilde{y})$ lies entirely above $q_{c}(\tilde{y})$, which means that corresponding modes are completely Landau damped. In all other cases $(0<\alpha<1)$, however, Landau damping will affect the modes only partially.

Let us now form the limit $n, W \rightarrow \infty$ where the ratio $n / W$ $=q_{n}(0)$ is kept fixed. In this regime it is well justified (see below) to assume that Landau damping is so strong as to suppress the mode locally in the regions $\left|\tilde{y}_{c}^{(n)}\right|<|\tilde{y}|<1$ in which it occurs, ${ }^{22}$ i.e., for $q_{n}(\tilde{y})>q_{c}(\tilde{y})$. The linewidth formula (37) then becomes

$$
\begin{aligned}
\frac{\Gamma_{n}}{\omega_{n}} \stackrel{n, W \rightarrow \infty}{\longrightarrow} \frac{q_{n}(0)}{2 \pi e^{* 2}} \\
\quad \times \frac{\int_{-\tilde{y}_{c}^{(n)}}^{\tilde{y}_{c}^{(n)}} d \tilde{y}\left(1-\tilde{y}^{2}\right)^{-1}\left|\operatorname{Im} f_{\mathrm{xcL}}^{h}\left(\varrho_{0}(\tilde{y}), \omega_{n}\right)\right|}{\int_{-\tilde{y}_{c}^{(n)}}^{\tilde{y}^{(n)}} d \tilde{y}\left(1-\tilde{y}^{2}\right)^{-1 / 2}} .
\end{aligned}
$$

To arrive at this relation, we have utilized the identities ${ }^{20}$

$$
\begin{gathered}
\frac{\partial U_{n-1}}{\partial \tilde{y}}=\frac{1}{1-\tilde{y}^{2}}\left[U_{n}-(n+1) T_{n}\right], \\
U_{n-1}^{2}=\frac{1}{1-\tilde{y}^{2}}\left(1-T_{n}^{2}\right) .
\end{gathered}
$$

Recognizing the fact that the $T_{n}$ become more and more rapidly oscillating between -1 and 1 for increasing $n$, we can replace them by their average value 0.5 .

Clearly, the simple notion of "local damping" will not be valid in the regime where both $n$ and $W$ are finite. Here, we need a more refined, nonlocal criterion to account for Landau damping. The idea is to perform a global Fourier analysis of the mode and to define a cutoff in momentum space rather that in real space. This procedure may be viewed as a "filter" to remove the large- $q$ components of the mode, which are the ones to be suppressed by Landau damping. In practice, we replace the Chebyshev polynomials $U_{n-1}(\tilde{y})$ in Eq. (33) by their convolution with the Fourier transform of a rectangular window in $q$ space:

$$
\bar{U}_{n-1}(\tilde{y})=\frac{1}{\pi} \int_{-1}^{1} d x U_{n-1}(x) \frac{\sin [\tilde{q} c, n(\tilde{y}-x)]}{\tilde{y}-x} .
$$

For a given width $2 W$ of the strip, $\tilde{q}_{c, n}$ is defined as $\tilde{q}_{c, n}$ $\equiv q_{n}\left(\tilde{y}_{c}^{(n)}\right) W$. The ratio between linewidth and mode frequency of the $n$th collective mode then reads

$$
\frac{\Gamma_{n}}{\omega_{n}}=\frac{q_{n}(0)}{2 \pi e^{* 2}} \frac{\int_{-1}^{1} d \tilde{y}\left(1-\tilde{y}^{2}\right)\left|\operatorname{Im} f_{\mathrm{xcL}}^{h}\left(\varrho_{0}(\tilde{y}), \omega_{n}\right)\right|\left[(1 / n)\left(\partial \bar{U}_{n-1}(\tilde{y}) / \partial \tilde{y}\right)\right]^{2}}{\int_{-1}^{1} d \tilde{y} \sqrt{1-\tilde{y}^{2}} \bar{U}_{n-1}^{2}(\tilde{y})} .
$$

In the limit $n, W \rightarrow \infty$ with $n / W$ fixed, $\bar{U}_{n-1}(\tilde{y})$ tends to $U_{n-1}(\tilde{y})$ for $|\tilde{y}|<\left|\tilde{y}_{c}^{(n)}\right|$ and zero otherwise, which a posteriori justifies the limit (44) of local damping.

\section{Numerical results and discussion}

Before applying the linewidth formulas (37), (44) and (48) to a concrete example, it is instructive to have a look at the homogeneous 2DEG. The long-wavelength limit of the random-phase approximation plasmon dispersion is well known: ${ }^{21,23}$

$$
\omega_{2 \mathrm{DEG}}(q)^{2}=\frac{2 \pi \bar{\varrho} e^{* 2}}{m^{*}} q
$$

where $\bar{\varrho}$ is the homogeneous $2 \mathrm{D}$ density and $q$ is the wave vector. The ratio between the linewidth of collective modes and the mode frequency is given (for small $q$ ) by

$$
\frac{\Gamma_{2 \mathrm{DEG}}}{\omega_{2 \mathrm{DEG}}}=\frac{q}{2 \pi e^{* 2}}\left|\operatorname{Im} f_{\mathrm{xc}}^{h}\left(\bar{\varrho}, \omega_{2 \mathrm{DEG}}\right)\right| .
$$




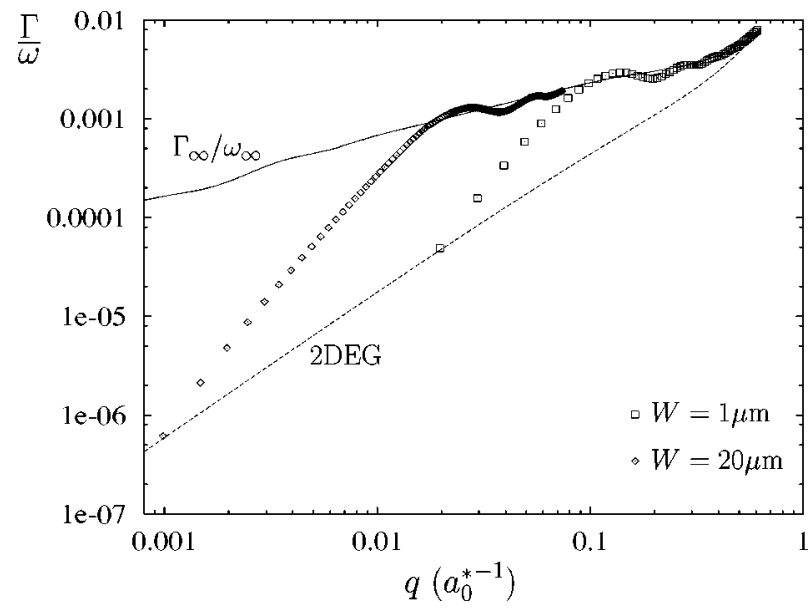

FIG. 2. Ratio of linewidth $\Gamma$ and mode frequency $\omega$ vs wave vector. Dashed line: $\Gamma_{2 \mathrm{DEG}} / \omega_{2 \mathrm{DEG}}$ for a homogeneous $2 \mathrm{DEG}$ of density $\bar{\varrho}=2 \times 10^{11} \mathrm{~cm}^{-2}$. Full line: limiting curve $\Gamma_{\infty} / \omega_{\infty}$ for an infinitely large quantum strip, with $\varrho_{0}(0)=\bar{\varrho}$ and fixed $q=n / W$. Symbols: $\Gamma_{n} / \omega_{n}$ vs $q_{n}=n / W$ for two different quantum strips with $\varrho_{0}(0)=\bar{\varrho}$ and widths $W=1 \mu \mathrm{m}$ (squares) and $W=20 \mu \mathrm{m}$ (diamonds). Each symbol denotes a collective mode with index $n$, starting with $n=2$.

Similar to the 3D case, ${ }^{6,15}$ this result follows from finding the zeros of the dielectric function in the long-wavelength limit. ${ }^{21}$ Note that we define the linewidth $\Gamma$ as twice the imaginary part of the mode frequency.

In the following, we shall compare the linewidths of collective modes in quantum strips with the plasmon linewidths in a homogeneous 2DEG. Density $\bar{\varrho}$ and wave vector $q$ in the 2DEG are taken to be the same as the equilibrium density $\varrho_{0}(0)$ and the local wave vector $q_{n}(0)=n / W$ in the center of the strips. The plasmon frequency (49) then exactly matches the corresponding mode frequency in the strip, Eq. (31). However, it will turn out that the imaginary parts of the mode frequencies are strikingly different.

To evaluate the homogeneous and inhomogeneous linewidth formulas, we furthermore need the imaginary part of $f_{\mathrm{xcL}}^{h}\left(\varrho_{0}, \omega\right)$ in two dimensions as input. For this we employ the parametrization by Nifosi, Conti, and Tosi, ${ }^{17}$ given for discrete values of $r_{s}$ and variable $\omega$, and use cubic splines to interpolate $f_{\mathrm{xcL}}^{h}$ for fixed $\omega_{n}$ and variable $r_{s} \geqslant 1 / \sqrt{\pi \bar{\varrho}}$. Note that the $q$ dependence of $f_{\mathrm{xcL}}^{h}$ has been neglected.

Our numerical results are summarized in Fig. 2, which shows $\Gamma / \omega$ versus wave vector for the homogeneous $2 \mathrm{DEG}$ and two different quantum strips. In the latter case, $q$ refers to the center of the strip, i.e., $q \equiv n / W$. As in the example treated in Sec. IV C, we consider here systems based on $\mathrm{GaAs} / \mathrm{Al}_{x} \mathrm{Ga}_{1-x}$ As with $\varrho_{0}(0)=\bar{\varrho}=2.0 \times 10^{11} \mathrm{~cm}^{-2}$.

The dashed line shows $\Gamma_{2 \mathrm{DEG}} / \omega_{2 \mathrm{DEG}}$ for the homogeneous system, up to a cutoff wave vector $q_{c}=0.614 a_{0}^{*-1}$. We find that $\Gamma_{2 \mathrm{DEG}} / \omega_{2 \mathrm{DEG}}$ grows as $q^{3 / 2}$ for small $q$. This is easily seen from Eq. (50) by noting that $f_{\text {xcL }}^{h}\left(\varrho_{0}, \omega\right)$ starts out linearly with $\omega$ (see Ref. 17), while from Eq. (49) we have $\omega \propto \sqrt{q}$.

The discrete symbols refer to $\Gamma_{n} / \omega_{n}$ for two quantum strips with different widths: $W=1 \mu \mathrm{m}$ (squares) and $W$
$=20 \mu \mathrm{m}$ (diamonds). The points represent the collective modes with increasing index $n=q W$. Let us first consider small values of $n$, for which Eq. (37) applies. For the lowest mode with nonvanishing linewidth $(n=2), \Gamma_{n} / \omega_{n}$ is in both cases only slightly higher than the corresponding $\Gamma_{2 \mathrm{DEG}} / \omega_{2 \mathrm{DEG}}$. In contrast to the latter, however, $\Gamma_{n} / \omega_{n}$ grows initially as $q^{3}$ and therefore reaches values that are considerably higher than $\Gamma_{2 \mathrm{DEG}} / \omega_{2 \mathrm{DEG}}$. Comparing Eqs. (37) and (50), we see that this enhancement is mainly due to the Chebyshev polynomials $U_{n-1}$, which have the form of sines and cosines for $\tilde{y} \approx 0$, see Eq. (40), but oscillate more and more rapidly as one moves away from the center of the strip, so that $\left(\partial U_{n-1} / \partial \tilde{y}\right)^{2}$ strongly increases. In addition to this, $\operatorname{Im} f_{\mathrm{xcL}}^{h}\left(\varrho_{0}(\tilde{y}), \omega_{n}\right)$ grows with decreasing density $\varrho_{0}(\tilde{y})$. On the other hand, the factor $\left(1-\tilde{y}^{2}\right)$ goes to zero for $\tilde{y} \rightarrow \pm 1$, but is too weak to suppress the enhancement over the homogeneous case.

After the initial rapid increase over the first few $n$, the modes start picking up Fourier components beyond the cutoff momentum, which have to be removed to account for Landau damping as discussed above, see Eq. (48). The $\Gamma_{n} / \omega_{n}$ for both strips then approach the limiting curve $\Gamma_{\infty} / \omega_{\infty}$ (see below). For $W=1 \mu \mathrm{m}$ this happens at around $n=10$, and for $W=20 \mu \mathrm{m}$ at around $n=40$. For the narrower strip, the highest mode below $q_{c}$ has the index $n$ $=62$. All higher modes will be completely Landau damped, as discussed in Sec. IV C. For the wider strip, only the first 150 modes have been indicated. The higher modes (up to $n$ $=1249$ ) are very densely spaced and all fall on the limiting curve.

Letting $W$ and $n$ approach infinity for a given $q=n / W$ and fixed $\varrho_{0}(0)$, we obtain the limit $\Gamma_{\infty} / \omega_{\infty}$ from Eq. (44), as shown by the full line. We note that $\Gamma_{\infty} / \omega_{\infty}$ grows as $\sqrt{q}$ for small $q$ and is almost three orders of magnitude above $\Gamma_{2 \mathrm{DEG}} / \omega_{2 \mathrm{DEG}}$ for $q=0.001 a_{0}^{*-1}$. As $q$ increases, the distance between the curves becomes less, and they merge at $q=q_{c}$. To understand this behavior, recall that $\left|\tilde{y}_{c}\right| \rightarrow 0$ for $q \rightarrow q_{c}$. In this limit one integrates in Eq. (44) only over a narrow stretch in the interior region of the strip where the density is practically uniform.

To summarize, the viscosity-induced linewidth of collective modes in finite quantum strips is found to be substantially higher than in the homogeneous 2DEG, in particular for small wave vectors. This enhancement is due to the strong inhomogeneity in the strips, and it persists even if we let their width become infinitely large. The explanation for this lies in the equilibrium density distribution (26), the shape of which scales with $y / W$ and is therefore invariant if the width of the strip changes. This means that the presence of the boundaries is always felt over the whole interior region of the quantum strip, regardless of its width. This striking feature can be traced back to the parabolic shape of the confining potential. A limiting process that for increasing width eventually leads to the homogeneous 2DEG would have to eliminate the influence of the boundaries. This may for instance be accomplished by letting the confining potential gradually approach a square-well form for large widths, thus arriving at a uniform equilibrium density distribution over the whole strip. 


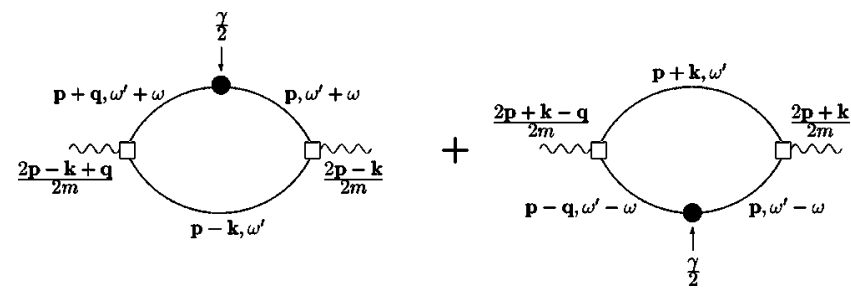

FIG. 3. First-order diagrams contributing to the paramagnetic part of the noninteracting current-current response function.

\section{CONCLUSION}

In this paper, we have proposed a simple way to calculate the linewidths of collective electronic excitations induced by damping through dynamical xc effects. The method is based on time-dependent current density-functional theory, and it includes exchange and correlation effects in terms of viscoelastic stresses in the electron fluid. The basic idea is to first calculate the collective modes using the adiabatic localdensity approximation. The latter accounts for single particle-hole production (Landau damping) only, i.e., highfrequency, long-wavelength modes will be undamped. The viscosity-induced damping effects are then added on perturbatively.

We have applied the formalism to calculate the linewidths of collective modes in $2 \mathrm{D}$ quantum strips. The advantage of these systems is that the mode profiles are known analytically through solution of the linearized classical equation of motion. It was found that the modes in the strips are much more strongly damped than the corresponding plasmon modes in a homogeneous 2DEG. For small wave vectors, we observed an order-of-magnitude enhancement over the 2DEG due to the inhomogeneity of the strips. As the regime of Landau damping is approached for growing wave vectors, the enhancement disappears. Contrary to expectation, we found that the behavior of the quantum strips with a parabolic confining potential does not approach that of a 2DEG if the width is increased to infinity.

\section{ACKNOWLEDGMENTS}

This work was supported by Research Board Grant No. RB 96-071 from the University of Missouri and by NSF Grant No. DMR-9706788. We thank Sergio Conti and Riccardo Nifosì for sending us their results on $f_{\mathrm{xcL}}^{h}$ in $2 \mathrm{D}$.

\section{APPENDIX}

In this appendix, we derive an explicit expression for the Kohn-Sham current-current response function $\chi_{\mathrm{KS}, \mu \nu}\left(\mathbf{r}, \mathbf{r}^{\prime}, \omega\right)$, see Eq. (3), valid for systems in which the unperturbed static density $\varrho_{0}(\mathbf{r})$ is slowly varying on the scales of the local $k_{F}^{-1}$ and $v_{F} / \omega$. Besides its importance in the present work, this formula for $\chi_{\mathrm{KS}, \mu \nu}$ could also be of use in the study of van der Waals interactions. ${ }^{24,25}$ For this reason we supply here some details of the derivation.

In order to obtain the coefficients of the gradient expansion of $\chi_{\mathrm{KS}, \mu \nu}$ up to second order in the gradients, we first consider an electron gas subject to a potential modulation $v_{0}(\mathbf{r})=\gamma \cos (\mathbf{q} \cdot \mathbf{r})$ that is both small and slowly varying. Thus,

$$
\varrho_{0}(\mathbf{r})=\bar{\varrho}+\gamma \chi^{h}(\mathbf{q}, 0) \cos (\mathbf{q} \cdot \mathbf{r}),
$$

where $q \ll k_{F}, \omega / v_{F}$ and $\gamma \ll 1$. Here, $\chi^{h}(\mathbf{q}, 0)$ is the static noninteracting response function, whose small- $q$ limit is given by ${ }^{15}-k_{F}^{2} /(3 \bar{\varrho} m)$. Let us now denote the second (i.e., the paramagnetic) part on the right-hand side of definition (3) by $R_{\mu \nu}\left(\mathbf{r}, \mathbf{r}^{\prime}, \omega\right)$. We introduce the momentum representation of $R_{\mu \nu}$ as

$$
\begin{aligned}
& R_{\mu \nu}(\mathbf{k}+s \mathbf{q}, \mathbf{k}, \omega) \\
& \quad=\int d^{3} r \int d^{3} r^{\prime} R_{\mu \nu}\left(\mathbf{r}, \mathbf{r}^{\prime}, \omega\right) e^{-i(\mathbf{k}+s \mathbf{q}) \mathbf{r}} e^{i \mathbf{k} \mathbf{r}^{\prime}},
\end{aligned}
$$

where $s$ is an integer. The inverse of this relation reads

$$
R_{\mu \nu}\left(\mathbf{r}, \mathbf{r}^{\prime}, \omega\right)=\sum_{s} \int d^{3} k R_{\mu \nu}(\mathbf{k}+s \mathbf{q}, \mathbf{k}, \omega) e^{i(\mathbf{k}+s \mathbf{q}) \mathbf{r}} e^{-i \mathbf{k} \mathbf{r}^{\prime}}
$$

To first order in $\gamma$, only those components of $R_{\mu \nu}$ with $s$ $=0, \pm 1$ are nonvanishing, and we have

$$
R_{\mu \nu}(\mathbf{k}, \mathbf{k}, \omega) \cong R_{\mu \nu}^{h}(\mathbf{k}, \omega)
$$

Here, $R_{\mu \nu}^{h}(\mathbf{k}, \omega)$ denotes the paramagnetic current response tensor of the homogeneous electron gas, which is given by ${ }^{12}$

$$
R_{\mu \nu}^{h}(\mathbf{k}, \omega)=\frac{\bar{\varrho} k_{F}^{2}}{5 m^{3} \omega^{2}}\left[2 k_{\mu} k_{\nu}+k^{2} \delta_{\mu \nu}\right]
$$

To calculate the off-diagonal contribution $R_{\mu \nu}(\mathbf{k}+\mathbf{q}, \mathbf{k}, \omega)$, we start with the expression

$$
\begin{aligned}
R_{\mu \nu}(\mathbf{k}+\mathbf{q}, \mathbf{k}, \omega)= & \frac{\gamma}{2 m^{2}} \sum_{\mathbf{p}, \omega^{\prime}}\left(\mathbf{p}-\frac{\mathbf{k}}{2}+\frac{\mathbf{q}}{2}\right)_{\mu}\left(\mathbf{p}-\frac{\mathbf{k}}{2}\right)_{\nu} \\
& \times G\left(\mathbf{p}-\mathbf{k}, \omega^{\prime}\right) G\left(\mathbf{p}, \omega+\omega^{\prime}\right) \\
& \times G\left(\mathbf{p}+\mathbf{q}, \omega+\omega^{\prime}\right) \\
& +(\mathbf{q} \rightarrow-\mathbf{q}, \mathbf{k} \rightarrow-\mathbf{k}, \omega \rightarrow-\omega),
\end{aligned}
$$

which follows from the first-order diagrams shown in Fig. 3 according to the usual rules of diagrammatic many-body theory. ${ }^{6} G$ denotes the free-particle Green's function:

$$
G(\mathbf{p}, \omega)=\left[\omega-\epsilon_{\mathbf{p}}-i \eta \operatorname{sign}\left(k_{F}-p\right)\right]^{-1}, \quad \eta=0^{+}
$$

where $\epsilon_{\mathbf{p}}=p^{2} / 2 m$. The frequency integral in Eq. (A6) is easily carried out using contour integration, and we obtain, after some rearrangement, 


$$
\begin{aligned}
R_{\mu \nu}(\mathbf{k}+\mathbf{q}, \mathbf{k}, \omega)= & \frac{\gamma}{m^{2}} \sum_{\mathbf{p}}\left(\mathbf{p}+\frac{\mathbf{k}+\mathbf{q}}{2}\right)_{\mu} \\
& \times\left(\mathbf{p}+\frac{\mathbf{k}}{2}\right) \frac{1}{\epsilon_{\mathbf{p}+\mathbf{k}+\mathbf{q}}-\epsilon_{\mathbf{p}+\mathbf{k}}} \\
& \times\left[\frac{\left(n_{\mathbf{p}+\mathbf{k}}-n_{\mathbf{p}}\right)\left(\boldsymbol{\epsilon}_{\mathbf{p}+\mathbf{k}}-\epsilon_{\mathbf{p}}\right)}{\omega^{2}+\left(\boldsymbol{\epsilon}_{\mathbf{p}+\mathbf{k}}-\boldsymbol{\epsilon}_{\mathbf{p}}\right)^{2}}\right. \\
& \left.-\frac{\left(n_{\mathbf{p}+\mathbf{k}+\mathbf{q}}-n_{\mathbf{p}}\right)\left(\boldsymbol{\epsilon}_{\mathbf{p}+\mathbf{k}+\mathbf{q}}-\epsilon_{\mathbf{p}}\right)}{\omega^{2}-\left(\epsilon_{\mathbf{p}+\mathbf{k}+\mathbf{q}}-\epsilon_{\mathbf{p}}\right)^{2}}\right],
\end{aligned}
$$

with $n_{\mathbf{p}}=\theta\left(k_{F}-p\right)$. In the limit of small $k$ and $q$ we can neglect $\left(\epsilon_{\mathbf{p}+\mathbf{k}}-\epsilon_{\mathbf{p}}\right)^{2}$ and $\left(\epsilon_{\mathbf{p}+\mathbf{k}+\mathbf{q}}-\epsilon_{\mathbf{p}}\right)^{2}$ with respect to $\omega$ in the denominators. Equation (A8) thus simplifies to

$$
\begin{aligned}
R_{\mu \nu}(\mathbf{k}+\mathbf{q}, \mathbf{k}, \omega) \cong & \frac{\gamma}{m^{2} \omega^{2}} \sum_{\mathbf{p}}\left(\mathbf{p}+\frac{\mathbf{k}+\mathbf{q}}{2}\right)_{\mu}\left(\mathbf{p}+\frac{\mathbf{k}}{2}\right)_{\nu} \\
& \times\left[\frac{\left(n_{\mathbf{p}+\mathbf{k}}-n_{\mathbf{p}}\right)\left(\boldsymbol{\epsilon}_{\mathbf{p}+\mathbf{k}}-\boldsymbol{\epsilon}_{\mathbf{p}}\right)}{\epsilon_{\mathbf{p}+\mathbf{k}+\mathbf{q}}-\boldsymbol{\epsilon}_{\mathbf{p}+\mathbf{k}}}\right. \\
& \left.-\frac{\left(n_{\mathbf{p}+\mathbf{k}+\mathbf{q}}-n_{\mathbf{p}}\right)\left(\boldsymbol{\epsilon}_{\mathbf{p}+\mathbf{k}+\mathbf{q}}-\epsilon_{\mathbf{p}}\right)}{\epsilon_{\mathbf{p}+\mathbf{k}+\mathbf{q}}-\boldsymbol{\epsilon}_{\mathbf{p}+\mathbf{k}}}\right]
\end{aligned}
$$

Expanding $n_{\mathbf{p}+\mathbf{k}}$ and $n_{\mathbf{p}+\mathbf{k}+\mathbf{q}}$ around $n_{\mathbf{p}}$, the terms in the square brackets reduce to

$$
\begin{aligned}
& -\frac{n_{\mathbf{p}}^{\prime}}{m}\left[\mathbf{k} \cdot \mathbf{p}+\frac{k^{2}}{2}+(\mathbf{k}+\mathbf{q}) \cdot \mathbf{p}+\frac{(\mathbf{k}+\mathbf{q})^{2}}{2}\right]+\frac{n_{\mathbf{p}}^{\prime \prime}}{2 m^{2}}\left\{(\mathbf{k} \cdot \mathbf{p})^{2}\right. \\
& \left.+[(\mathbf{k}+\mathbf{q}) \cdot \mathbf{p}]^{2}+(\mathbf{k} \cdot \mathbf{p})[(\mathbf{k}+\mathbf{q}) \cdot \mathbf{p}]\right\}
\end{aligned}
$$

The momentum integration can now be carried out, and after some lengthy algebra we end up with

$$
\begin{aligned}
R_{\mu \nu}(\mathbf{k}+\mathbf{q}, \mathbf{k}, \omega)= & -\frac{\gamma \bar{\varrho}}{2 m^{2} \omega^{2}} \\
& \times\left[q_{\mu} q_{\nu}+\mathbf{k} \cdot(\mathbf{k}+\mathbf{q}) \delta_{\mu \nu}+2 k_{\mu}\left(k_{\nu}+q_{\nu}\right)\right] .
\end{aligned}
$$

We now insert the zero- and first-order contributions (A5) and (A11) into Eq. (A3) to transform back into coordinate space. From the resulting expression, we can identify the coefficients of the gradient expansion of $\chi_{\mathrm{KS}, \mu \nu}$. The final result is

$$
\begin{aligned}
\chi_{\mathrm{KS}, \mu \nu}\left(\mathbf{r}, \mathbf{r}^{\prime}, \omega\right)= & \frac{\varrho_{0}(\mathbf{r})}{m} \delta\left(\mathbf{r}-\mathbf{r}^{\prime}\right) \delta_{\mu \nu}-\frac{\varrho_{0}(\mathbf{r}) k_{F}^{2}}{5 m^{3} \omega^{2}}\left(2 \nabla_{\mu} \nabla_{\nu}\right. \\
& \left.+\nabla^{2} \delta_{\mu \nu}\right) \delta\left(\mathbf{r}-\mathbf{r}^{\prime}\right)-\frac{k_{F}^{2}}{3 m^{3} \omega^{2}} \delta\left(\mathbf{r}-\mathbf{r}^{\prime}\right) \\
& \times\left[\nabla_{\mu} \nabla_{\nu}-\left(\nabla \cdot \nabla^{\prime} \delta_{\mu \nu}+2 \nabla_{\mu}^{\prime} \nabla_{\nu}\right)\right] \varrho_{0}(\mathbf{r}) .
\end{aligned}
$$

From this, the inverse, correct up to second order in the gradients, is easily obtained as

$$
\begin{aligned}
\chi_{\mathrm{KS}, \mu \nu}^{-1}\left(\mathbf{r}, \mathbf{r}^{\prime}, \omega\right)= & \frac{m^{2}}{\varrho_{0}^{2}(\mathbf{r})}\left\{\frac{\varrho_{0}(\mathbf{r})}{m} \delta\left(\mathbf{r}-\mathbf{r}^{\prime}\right) \delta_{\mu \nu}\right. \\
& +\frac{\varrho_{0}(\mathbf{r}) k_{F}^{2}}{5 m^{3} \omega^{2}}\left(2 \nabla_{\mu} \nabla_{\nu}+\nabla^{2} \delta_{\mu \nu}\right) \delta\left(\mathbf{r}-\mathbf{r}^{\prime}\right) \\
& +\frac{k_{F}^{2}}{3 m^{3} \omega^{2}} \delta\left(\mathbf{r}-\mathbf{r}^{\prime}\right)\left[\nabla_{\mu} \nabla_{\nu}-\left(\nabla \cdot \nabla^{\prime} \delta_{\mu \nu}\right.\right. \\
& \left.\left.\left.+2 \nabla_{\mu}^{\prime} \nabla_{\nu}\right)\right] \varrho_{0}(\mathbf{r})\right\} .
\end{aligned}
$$

${ }^{1}$ See, for example, D. Heitmann, in Physics of Nanostructures, edited by J. H. Davies and A. R. Long (IOP Publishing, London, 1992), and references therein.

${ }^{2}$ P. R. Pinsukanjana, E. G. Gwinn, J. F. Dobson, E. L. Yuh, N. G. Asmar, M. Sundaram, and A. C. Gossard, Phys. Rev. B 46, 7284 (1992); A. Wixforth, M. Kaloudis, C. Rocke, K. Ensslin, M. Sundaram, J. H. English, and A. C. Gossard, Semicond. Sci. Technol. 9, 215 (1994); E. L. Yuh, E. G. Gwinn, P. R. Pinsukanjana, W. L. Schaich, K. L. Campman, P. F. Hopkins, and A. C. Gossard, Phys. Rev. B 54, 11467 (1996).

${ }^{3}$ K. Craig, C. L. Felix, J. N. Heyman, A. G. Markelz, M. S. Sherwin, K. L. Campman, P. F. Hopkins, and A. C. Gossard, Semicond. Sci. Technol. 9, 627 (1994).

${ }^{4}$ J. N. Heyman, K. Craig, B. Galdrikian, M. S. Sherwin, K. Campman, P. F. Hopkins, S. Fafard, and A. C. Gossard, Phys. Rev. Lett. 72, 2183 (1994); J. N. Heyman, K. Unterrainer, K. Craig, B. Galdrikian, M. S. Sherwin, K. Campman, P. F. Hopkins, and A. C. Gossard, ibid. 74, 2682 (1995); M. S. Sherwin, K. Craig, B. Galdrikian, J. Heyman, A. Markelz, K. Campman, S. Fafard, P. F. Hopkins, and A. Gossard, Physica D 83, 229 (1995); J. B.
Williams, K. Craig, M. S. Sherwin, K. Campman, and A. C. Gossard, in MSS8 Conference Proceedings [Physica B (to be published)].

${ }^{5}$ D. Pines and P. Nozières, The Theory of Quantum Liquids (W. A. Benjamin, New York, 1966).

${ }^{6}$ A. L. Fetter and J. D. Walecka, Quantum Theory of ManyParticle Systems (McGraw-Hill, New York, 1971).

${ }^{7}$ E. K. U. Gross and W. Kohn, Phys. Rev. Lett. 55, 2850 (1985); Erratum, ibid. 57, 923 (1986).

${ }^{8}$ E. K. U. Gross and W. Kohn, Adv. Quantum Chem. 21, 255 (1990); E. K. U. Gross, J. F. Dobson, and M. Petersilka, in Topics in Current Chemistry, edited by R. F. Nalewajski (Springer, Berlin, 1996).

${ }^{9}$ J. F. Dobson and G. H. Harris, J. Phys. C 21, L729 (1988).

${ }^{10}$ J. F. Dobson, Phys. Rev. B 46, 10163 (1992).

${ }^{11}$ G. Vignale and W. Kohn, Phys. Rev. Lett. 77, 2037 (1996).

${ }^{12} \mathrm{G}$. Vignale and W. Kohn, in Electronic Density Functional Theory, edited by J. F. Dobson, G. Vignale, and M. K. Das (Plenum Press, New York, 1998).

${ }^{13}$ G. Vignale, C. A. Ullrich, and S. Conti, Phys. Rev. Lett. 79, 4878 (1997). 
${ }^{14}$ W. L. Schaich, M. R. Geller, and G. Vignale, Phys. Rev. B 53, 13016 (1996).

${ }^{15}$ K. S. Singwi and M. P. Tosi, in Solid State Physics, edited by H. Ehrenreich, F. Seitz, and D. Turnbull (Academic, New York, 1981), Vol. 36, p. 177.

${ }^{16}$ H. M. Böhm, S. Conti, and M. P. Tosi, J. Phys.: Condens. Matter 8, 781 (1996); S. Conti, R. Nifosi, and M. P. Tosi, ibid. 9, L475 (1997).

${ }^{17}$ R. Nifosi, S. Conti, and M. P. Tosi, Phys Rev. B (to be published).

${ }^{18}$ L. D. Landau and E. Lifshitz, Mechanics of Fluids, 2nd ed., course of Theoretical Physics Vol. 6 (Pergamon Press, Oxford, 1987).

${ }^{19}$ V. B. Shikin, T. Demel, and D. Heitmann, Zh. Eksp. Teor. Fiz. 96, 1406 (1989) [Sov. Phys. JETP 69, 797 (1989)].

${ }^{20}$ M. Abramowitz and I. A. Stegun, Handbook of Mathematical
Functions (Dover, New York, 1970).

${ }^{21}$ A. Czachor, A. Holas, S. R. Sharma, and K. S. Singwi, Phys. Rev. B 25, 2144 (1982).

${ }^{22}$ A simple estimate of the strength of Landau damping of smallwavelength modes is obtained using the $2 \mathrm{D}$ Lindhard function $\chi_{0}(q, \omega)$. Comparing $\left(m * \omega_{n}^{2} / \varrho_{0} q_{n}^{2}\right)\left|\operatorname{Im} \chi_{0}\left(q_{n}, \omega_{n}\right)\right| \quad$ with $\left(q_{n} / 2 \pi e^{* 2}\right)\left|\operatorname{Im} f_{\mathrm{xcL}}^{h}\left(\omega_{n}\right)\right|$ [see Eq. (7.5) of Ref. 14] we find that Landau damping is one to two orders of magnitude higher than $\mathrm{xc}$ damping in the regions where $\chi_{0}(q, \omega)$ is nonzero, leading to lifetimes of order of the period of the plasmon oscillations.

${ }^{23}$ F. Stern, Phys. Rev. Lett. 18, 546 (1967).

${ }^{24}$ J. F. Dobson and B. P. Dinte, Phys. Rev. Lett. 76, 1780 (1996).

${ }^{25}$ J. F. Dobson, B. P. Dinte, and J. Wang, in Electronic Density Functional Theory, edited by J. F. Dobson, G. Vignale, and M. K. Das (Plenum Press, New York, 1998). 